\title{
Study of The Spectrum of Traumatic Cataract Cases in A Tertiary Eye Hospital
}

\author{
Beena $\mathrm{R}^{1}$, Jeyalatha $\mathrm{D}^{2}$ \\ $\left({ }^{1,2}\right.$ Assistant Professor, Dept Of Ophthalmology, Kanyakumari Govt Medical College, Asaripallam, Dr. MGR \\ Medical University, Chennai, India )
}

\begin{abstract}
Backgrouund: Trauma is a leading cause of monocular blindness in the younger age group throughout the world.It is also a cause for significant morbidity.The morbidity becomes more compounded in the rural areas due to ignorance and lack of access to health facilities. Type of injury, preoperative, intraoperative and postoperative complications determine the visual prognosis. Assessment of the nature and extent of injuries will prepare the surgeon to deal with the eventualities arising intraoperatively and offer a better visual prognosis.

Aim and objective:To assess the demographic profile,age of patients presenting with traumatic cataract.To evaluate the nature of injuries, factors and complications which determine the visual prognosis.

Materials and Methods: The study is Hospital based, prospective study carried out for 25 months in the department of ophthalmology,Government Rajaji Hospital Madurai between August 2005 and September 2006.Detailed history regarding the demographic profile, the type,nature of injurywere collected. estimation of visal acuity, anterior segment examination, slit lamp \& fundus examination, intraocularpressure, intraoperative \&postoperative complications both immediate and delayed were noted to assess the visual prognosis.

Results: data was analysed using SSPA and the results were tabulated based on age,sex,nature of injury,time of onset, type of cataract, visual acuity, preoperative, intaoperative and postoperative complications

Conclusion: Traumatic cataract is a distinct entity associated with a poor visual prognosis compared to age related cataract.The outcome being determined bythe nature of injury and associated complications due to injury.

Proper education, timely attention and prudent management is essential in these cases to provide a reasonable visual acuity
\end{abstract}

Keywords: traumatic cataract,visualacuity,postoperative,blunt,penetrating injury

\section{Introduction}

Cataract literally meaning a downrushing or waterfall indicates a loss of transparency of the lens of the eye or its capsule. Cataract is the leading cause of preventable blindness in the world \& cataract extraction with intraocular lens(IOL) implantation is perhaps the most effective surgical procedure in all of medicine. Age related cataracts are a very common cause of visual impairment in older adults. However cataracts can occur in the young either congenitally, as a result of trauma, due to other systemic conditions or as a result of drug intake. Though originally reported by Ezra, Pioneering work in traumatic cataract has been done by Vogt with valuable contribution by Koby (1930), Lugli (1935) and Davidson (1940). The aim of the study is

1. To find out the demographic profile of patients, the characteristics of trauma and the incidence of traumatic cataract in various age groups.

2. To analyse the pre operative, intra operative and post operative complications associated with trauma.

3. To assess the factors influencing the visual prognosis in traumatic cataract.

\section{Materials And Methods}

This study was conducted at the Department of Ophthalmology, Government Rajaji Hospital, Madurai Medical College from August 2005 to Sept 2007 for a period of 25 months with a follow up ranging from 2 months to 1 year. All the patients presenting to our outpatient department, either on their own or on being referred were included in this study.The patients were registered, history was elicited they were examined thoroughly and then admitted in the wards. The total number of patients included in this study was 64 . Examination :After an initial examination using torch light, pupillary reaction was noted. Vision was recorded. Recording of intra ocular tension was done with applanation/ Schiotz where possible. Slit lamp examination was done to assess the type of corneal injury, associated iridocyclitis, foreign body / lens matter, hyphaema, hypopyon in anterior chamber. Iridodonesis, Iridodialysis, Iris hole, sphincter tear, posterior synechiae were looked for. Type of traumatic cataract subluxation of lens, evidence of vitritis was noted. Dilated fundus examination was done where possible by both Direct and Indirect ophthalmoscopy. Macular function tests like 
projection of light, colour perception were done.X ray orbit to rule out associated orbital fractures and intraocular foreign body was done. B -scan was done to rule out posterior segment pathology.Patients with documented history of trauma where the nature of injury or object of injury was known, patients with uninvolved posterior segment were included in the study.Patients with severe infection, patients with posterior segment pathology as documented by B-scan, fundus examination and patients with Intra ocular FB were excluded in the study.

Medical Treatment : Cases of Traumatic Cataract associated with an entry wound, corneal tear were treated with Oral Ciprofloxacin $500 \mathrm{mg}$ bid ,oral NSAIDS in addition to topical antibiotics. Cases with associated iridocyclitis were treated with topical and systemic steroids,.glaucoma was treated with anti glaucoma medications.

Anaesthesia : Most of the adults were given peribulbar block with facial Block while children and adults with systemic complications were given general anesthesia. Surgery :ECCE, SICS, or Phacoemulsification with PCIOL was done depending on the Surgeon's choice. Those patients with a corneal or corneo scleral tear requiring suturing were subjected to corneal tear suturing and excision of prolapsed iris in the first stage. Cataract surgery was done in the $2^{\text {nd }}$ stage. Patients with severe iridocyclitis were treated with steroids and taken up for surgery after 3 months of quiescence.

Post Operative Management :All patients were treated with antibioticsteroid eyedrops hourly, homatropine eyedrops t.i.d,oral NSAIDS. Postoperative iridocyclitis was treated with $1 \%$ prednisolone acetate eyedrops and atropine eyedrops.

Post Operative follow up : Patients were examined every day for first 4 days, every wee $\mathrm{k}$ for 1 month and every fortnight until $2^{\text {nd }}$ month. Refractive correction was prescribed at the end of $2^{\text {nd }}$ month.Patients with posterior capsule opacification were subjected to NdYAG after 6 months - 1year. Routine ocular examination was done for subsequent follow up.

\section{Results}

The total number of cases analysed under this study was 64 . The sex incidence of the patients was as follows.Sex incidence:Males 43(68.25\%) and Females 21(32.18\%) were affected.The urban - rural divide in our study was as follows.Urban $15(23.43 \%)$, Rural $49(76.56 \%)$. The patients presenting to our hospital are mostly from a rural agrarian society because Madurai is surrounded by many villages.

Age Distribution :

\begin{tabular}{|c|c|c|}
\hline Age & No.of patients & Incidence $\%$ \\
\hline $1-20$ & 17 & 26.5 \\
\hline $20-40$ & 24 & 37.5 \\
\hline $40-60$ & 18 & 28.5 \\
\hline 60 yrs and above & 5 & 17 \\
\hline
\end{tabular}

The majority of the cases involved in our study was in the 20-60 years age group $(65.62 \%)$. This could be due to the fact that most are agricultural workers and the elderly continue to work to make ends meet.The median age in our study was 38 years whereas in the Croation study it was 25 years.

Laterality: Incidence of trauma to the right eye was marginally more than the left.OD $33(51.5 \%) \& O S$

Type of Injury : Regarding the type of injury, Penetrating injury was more common accounting for 41 cases $(64.06 \%)$ and Blunt injuries in $23(35.93 \%)$. Thorn and stick being the most common objects of injury. In the East African study (3) of predominantly pediatric age group, thorn and stick were the commonest. Whereas in our pediatric population, cracker injury was common. In the Croation study cracker injury was the commonest (17).

Time of Onset :The time of onset ranged from 1 day being the earliest to 6 years being the longest duration. $<1$ week $25(39.06 \%) ;<2$ weeks $13(20.31) ;<2$ months $8(12.5 \%) ;<6$ months $9(14.06 \%) ;<1$ yr $5(7.8 \%)>1 \mathrm{yr} 4(6.25 \%)$

Pre Operative Visual Acuity : Pre operative visual acuity (VA) ranged from PL to > 6/60

\begin{tabular}{|c|c|c|}
\hline Pre operative Visual Acuity & No.of patients & Incidence \% \\
\hline PL & 17 & 26.56 \\
\hline HM & 19 & 29.68 \\
\hline CFCF & 9 & 14.06 \\
\hline $1 / 60-3 / 60$ & 8 & 12.5 \\
\hline$>6 / 60$ & 11 & 17.18 \\
\hline
\end{tabular}




\section{Pre operative Complications :}

The various preoperative complications encountered are classified as below.

\begin{tabular}{|c|l|c|c|}
\hline Site & \multicolumn{1}{|c|}{ Nature of Injury } & No.of patients & Incidence $\%$ \\
\hline Cornea & Corneal opacity & 26 & 40.62 \\
\hline & Self sealed corneal tears & 9 & 14.06 \\
\hline & $\begin{array}{l}\text { Tears requiring primary } \\
\text { suturing }\end{array}$ & 6 & 9.37 \\
\hline AC & Hyphaema & 3 & 4.68 \\
\hline & Lens matter in AC & 2 & 3.12 \\
\hline & Shallow AC & 18 & 28.12 \\
\hline & Irregular AC & 11 & 17.18 \\
\hline & Prolapse & 3 & 4.68 \\
\hline Iris & Iridocyclitis & 13 & 20.31 \\
\hline & Iridodialysis & 2 & 3.12 \\
\hline & Iridodonesis & 4 & 6.25 \\
\hline & Sphincter tear & 3 & 4.68 \\
\hline Lens & Subluxation & 4 & 6.25 \\
\hline Capsule & Primary PCO & 3 & 4.68 \\
\hline Vitreous & In front of lens & 1 & 1.56 \\
\hline Glaucoma & Lens Induced glaucoma & 9 & 14.06 \\
\hline
\end{tabular}

Glaucoma was predominantly a secondary angle closure glaucoma ie. Phacomorphic glaucoma due to intumescent cataract in 6 cases and anterior subluxation in 1 case while it was due to lens matter in AC and iridocyclitis in 2 cases.

Type of Cataract Surgery : Of the total 64 cases, 6 cases with corneal / corneoscleral tear which required primary suturing including 3 cases with iris prolapse, suturing of the tear was done primarily followed by a secondary cataract extraction later. ECCE with PCIOL either as primary or secondary procedure was done in 42 cases. ECCE with ACIOL was done in 1 case with traumatic subluxation of lens.ECCE only in 6 cases. Phacoemulsification with PCIOL in 2 cases and SICS with PCIOL in 9 cases. Trabeculectomy was done in 4 cases of phacomorphic glaucoma

Post operative Complications :

\begin{tabular}{|c|c|c|}
\hline Early Complications & No.of patients & Incidence $\%$ \\
\hline Iridocyclitis & 17 & 26.56 \\
\hline Striate keratopathy & 11 & 17.18 \\
\hline Corneal edema & 3 & 4.68 \\
\hline Shallow AC & 1 & 1.56 \\
\hline Cortex & 2 & 3.12 \\
\hline Hyphaema & 2 & 3.12 \\
\hline Vitritis & 2 & 3.12 \\
\hline Vitreous haemorrhage & 1 & 1.56 \\
\hline Increased IOP & 1 & 1.56 \\
\hline Iris prolapse & 1 & 1.56 \\
\hline
\end{tabular}

PCO was the predominant late complication in the study, accounting for 13 cases (20.31\%). This was also similar to the above mentioned studies

Late Complications :

\begin{tabular}{|c|c|c|}
\hline Complications & No.of patients & Incidence $\%$ \\
\hline PCO & 13 & 20.31 \\
\hline Pupillary capture & 4 & 6.25 \\
\hline IOL decentration & 2 & 3.14 \\
\hline CME & 3 & 4.68 \\
\hline
\end{tabular}

Post Operative Visual Acuity : The post operative visual acuity was recorded unaided at 3 days and later after 2 months the full refractive correction was given. During subsequent follow up, after 6 months -1 year YAG capsulotomy was done if PCO was significantly impairing vision.

\begin{tabular}{|c|c|c|}
\hline Post op. Vision unaided at 3 days & No.of patients & Incidence \% \\
\hline $6 / 6$ & 0 & 0 \\
\hline $6 / 9$ & 4 & 6.25 \\
\hline $6 / 12$ & 5 & 7.8 \\
\hline $6 / 18$ & 3 & 4.68 \\
\hline $6 / 24$ & 4 & 6.25 \\
\hline $6 / 36$ & 8 & 12.5 \\
\hline $6 / 60$ & 13 & 20.31 \\
\hline$<6 / 60$ & 27 & 42.18 \\
\hline
\end{tabular}




\begin{tabular}{|c|c|c|}
\hline Post op. Vision aided at 2 months & No.of patients & Incidence \% \\
\hline $6 / 6 \mathrm{P}$ & 3 & 5.26 \\
\hline $6 / 9$ & 9 & 15.78 \\
\hline $6 / 12$ & 10 & 17.54 \\
\hline $6 / 18$ & 6 & 10.52 \\
\hline $6 / 24$ & 5 & 8.77 \\
\hline $6 / 36$ & 5 & 8.77 \\
\hline $6 / 60$ & 6 & 10.52 \\
\hline$<6 / 60$ & 13 & 22.80 \\
\hline
\end{tabular}

6 patients were lost to follow up whereas vision was not recorded in 1 patient, as the patient had psychiatric manifestations. Out of the 57 cases followed up, the vision was better than 6/18 in 28 cases $49.12 \%$. The vision was between 6/24-6/36 in 10 cases with post operative complications and astigmatism. 19 patients had vision $\leq$ 6/60.The causes for poor vision were aphakia with vitreous loss during surgery in 3 cases.In 9 cases because of the primary corneal injury, which was either sutured or self sealed and in the pupillary area they had reduced vision. 2 cases had PCO.1vitreous haemorrhage, 1 decentration of IOL. In 3 cases the decrease in vision was due to post operative cystoid macular edema.

\section{Discussion}

In this study, a total of 64 cases of traumatic cataract with or without other associated injuries were studied. A look at the statistics reveals that the population involved was predominantly rural based population (76.56\%) and that males $(68.25 \%)$ were more commonly affected than females $(32.18 \%)$. The age incidence was slightly in the higher age group of 20-60 yrs with 42 cases $(65.62 \%)$. This was slightly in the higher range than other studies probably because of our predominantly agrarian population, where the elderly continued to work. The females involved also were farm hands or used to collect fire wood.Regarding laterality the right eye $(51.5 \%)$ was marginally involved more than the left eye (48.43\%).Out of the types of injuries, penetrating injury was definitely more commonly seen (ie.) in 41 cases $(64.06 \%)$ compared to blunt injuries (35.93\%). Stick was the commonest object causing injury, being responsible in 17 cases $(26.56 \%)$ of both penetrating and blunt injuries followed by thorn in 15 cases $(23.43 \%)$. The time of onset varied from one day being the earliest to 6 years being the oldest. Most of the penetrating injuries had an acute onset.

The preoperative visual acuity was HM or worse in 36 cases $(56.24 \%)$. The initial vision was better than $6 / 60$ only in 11 cases $(17.18 \%)$. The pre operative complications ranged from corneal tears requiring primary suturing in 6 cases $(9.37 \%)$ to self sealed corneal tears in 9 cases $(14.06 \%)$, Corneal opacities / wound of entry in 26 cases $(40.62 \%)$. The other salient pre operative complications were iridocyclitis in 13 cases (20.31\%), subluxation in 4 cases $(6.25 \%)$ and glaucoma either phacomorphic or lens matter induced in 9 cases.(14.06\%). Intra operative complications in the form of PC rent occurred in 3 cases (4.68\%) whereas 7 cases had pre existing PC rent. 8 cases were left aphakic whereas an ACIOL was implanted in 1 case. PCIOC was implanted in sulcus in one case. All the types of Cataract Surgery right from Phaco emulsification with PCIOL, SICS with PCIOL, ECCE with PCIOL and Combined surgery with PCIOL were done. ECCE with PCIOL was done in 42 cases which constituted (65.62\%). ECCE with ACIOL in 1 case (1.56\%). SICS with PCIOL in $9(14.06 \%)$, phacoemulsification with PCIOL in 2 cases $(3.12 \%)$. ECCE only in 6 cases $(9.37 \%)$. ECCE with trabeculectomy in 1 case $(1.56 \%)$, SICS with trabeculectomy in 1 case $(1.56 \%)$ and ECCE with trabeculectomy with PCIOL in 2 cases $(3.12 \%)$.. The type of IOL used was a PMMA lens and anterior vitrectomy by open sky method was done in all cases with PC rent.

The commonest early post operative complication was iridocyclitis, being present in 17 cases (26.56\%) and the commonest late post operative complication was posterior capsule opacification, present in 13 cases $(20.31 \%)$.

The unaided post operative visual acuity was $6 / 18$ or better in 12 cases $(18.79 \%)$. Between 6/24-6/60 in 25 cases $(39.06 \%)$ and $<6 / 60$ in 27 cases $(42.18 \%)$. However post operative best corrected visual acuity at 2 months was $6 / 18$ or better in 28 cases ie. $49.12 \%$, between 6/24-6/36 in 10 cases $(17.54 \%$ ) with post operative complications and only in 19 patients was the vision $\leq 6 / 60$ (29.68\%). These figures are for the 57 patients who returned for follow up. 6 patients were lost to follow up and 1 patient had psychiatric disturbances, so vision could not be recorded. Vision improved in 7 patients (12.28\%) with post operative YAG capsulotomy after 6 months -1 year and the average visual acuity following this ranged between 6/18-6/24. Visual acuity ranged between $<6 / 60-3 / 60$ in 6 cases and $<3 / 60$ in 7 cases $(12.28 \%)$ while it was $6 / 60$ in 6 cases $(10.52 \%)$. The reasons for poor vision were aphakia with vitreous loss during surgery in 3 patients $(5.26 \%)$. Corneal tear either repaired primarily or self healed esp. opposite pupillary area in 9 patients $(15.78 \%)$. Cystoid macular edema occurred in 3 cases. 2 patients had thick PCO but were in the early post operative period and hence were not subjected to YAG. 1 patient had vitreous haemorrhage and vitritis and was referred to a higher centre, while another had decentration of the lens. 


\section{Conclusion}

1. Males were comparatively affected more than females

2. A higher age group was affected due to the rural agrarian nature of population

3. Penetrating injuries were more common than blunt injuries. Stick \& thorn were the commonest objects

4. None of the industrial workers were wearing protective glasses at the time of injury.

5. There was a delay in obtaining medical attention due to the predominant illiterate population.

6. Intumescent cataract, lacerated and Rosette cataract were the commonest cataracts encountered.

7. Cases with corneal tear requiring suturing especially were done as a two stage procedures.

8. Penetrating injuries had worser outcome than blunt injuries.

9. ECCE with PCIOL, SICS with PCIOL or Phacoemulsification with PCIOL were used where appropriate depending on the clinical picture and all were satisfactory for the management of the traumatic cataract.

10. Iridocyclitis was the most common early post operative complication and posterior capsular opacification the commonest late post operative complications.

11. Visual acuity was $6 / 18$ or better in $49 \%$ of the cases after treatment.

12. The visual prognosis in most cases depended on pre operative complications, involving the cornea and on intra operative complications like posterior capsular rupture and post operative complications like posterior capsular opacification.

\section{References}

[1]. $\quad$ IJO volume 48 (3) Sept 2000 - Management of anterior segment penetrating injuries with traumatic cataract by pentagon approach in paediatric age group - Dr. Jitendra KS Parihar, Dr. Radha Govind Dash, Dr. Devender P Vats \& Coauthors.

[2]. Ophthalmology volume (vol) : 108(2) $2001 \mathrm{Feb}$. Successful rehabilitation of children with Traumatic Corneal laceration and cataract

[3]. J. cataract and refractive surgery vol 27 (12) Dec. IOL implantation for traumatic cataract in children in East Africa

[4]. J. Cataract refractive surgery volume 27(9) 2001 sept. Inaccuracy of IOL power calculation after traumatic corneal laceration and cataract.

[5]. Ophthalmology vol 110(3) 2003 March - Multifocal IOL in patient's with traumatic cataract

[6]. J. Cataract Refractive surgery - vol 28, 2002 Oct.

[7]. Arch ophthal. Volume 121(2) $2003 \mathrm{Feb}$

[8]. J. Cataract \& Refractive surgery vol 31 August 2005

[9]. J. Cataract Refractive surgery vol 26 (11) 2000 Nov.

[10]. American Journal of ophthalmology vol 130 (3) 2000 September

[11]. Eye Vol : 15 (1) $2001 \mathrm{Feb}$

[12]. J. Cataract and Refractive Surgery vol 29 (8) 2003 Aug

[13]. Eye vol : 20 (8) 2006 Aug.

[14]. J. Cataract Refractive Surgery vol. 27 (8) 2001 Aug.

[15]. Use of intra ocular lenses in children with traumatic cataract in South India. Michiel Eckstein, P. Vijayalakshmi, Milind Killedar, Clare Gilbert, Allen foster. Aravind Eye Hosital, Madurai in collaboration with Dept. of Preventive ophthalmology, Institute of ophthalmology, London.

[16]. Cataract and Refractive surgery today - June 2004

[17]. Croation Medical Journal 45 (3) : $310-313,2004$

[18]. Intraocular lens implantation in traumatic cataract, JK Practitioner, 1998 Jan - March ; 5(1) 41-43

[19]. Blum M, Tetz MR, Greiner C, Voelcker HE. Treatment of traumatic cataracts J. Cataract Refractive Surgery $1996 ; 22 ; 342-6$

[20]. Gain P, Thuret G, Maugery J. Management of traumatic cataracts J. Fr Ophthalmology, $2003 ; 26: 512-20$.

[21]. Gupta AK, Grover AK, Gurha N. Traumatic cataract Surgery with intra ocular lens implantation in children. J paediatric ophthalmology and strabismus $1992 ; 29: 73-8$

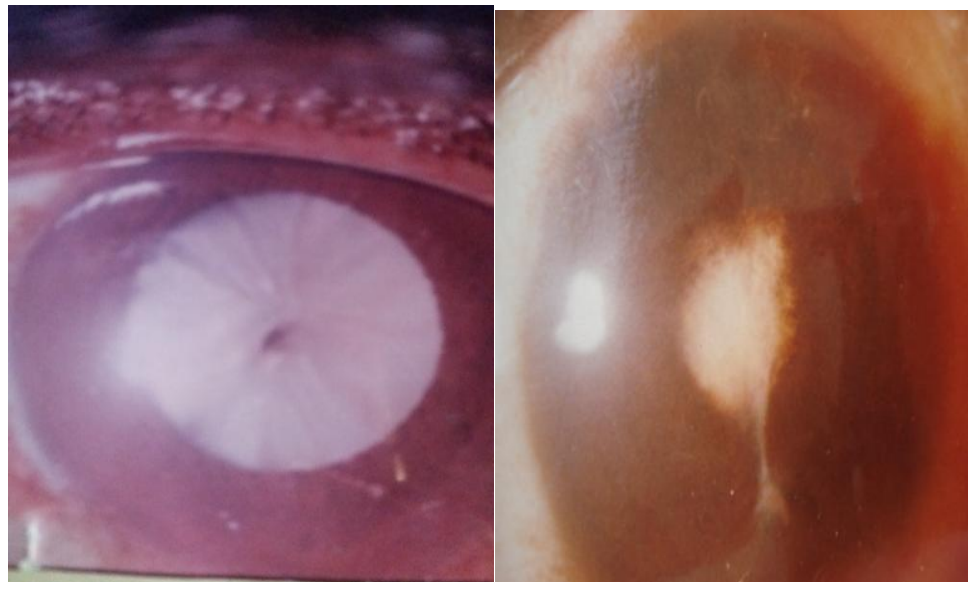




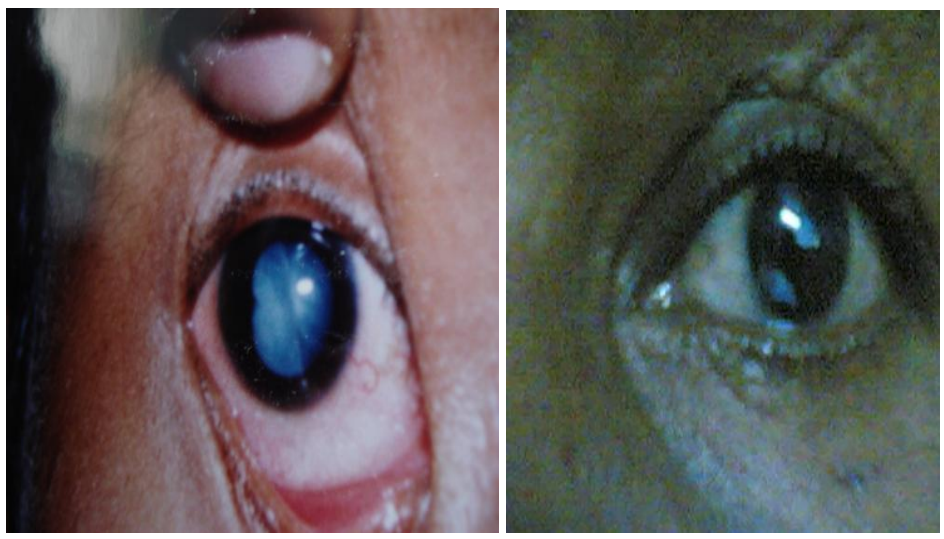

